\title{
SSRIs associated with fracture risk
}

Selective serotonin reuptake inhibitors (SSRIs) are associated with a significantly increased risk of fracture, suggests a new meta-analysis.

SSRIs are the most frequently prescribed agents for first-line management of depression. Previous studies investigating the link between use of this class of antidepressants and fracture risk have yielded inconsistent results. To clarify whether such an association exists, Qing Wu and colleagues performed a meta-analysis of cohort and case-control studies in which the occurrence of fractures and/or BMD values were compared between patients receiving or not receiving an SSRI.

Of the 13 studies meeting the inclusion criteria, the occurrence of fractures was reported in 11 studies, BMD in one study and both of these outcomes were reported in the remaining study.

The risk of fracture was significantly increased in patients taking SSRIs (relative risk 1.72 , 95\% CI 1.51-1.95, $P<0.001$ ). This association was independent of depression or BMD. "These findings suggest that the effect of SSRI treatment on risk of fracture may be through an increased propensity for falls," says Wu. "Therefore, patients taking SSRIs should be targeted for fall prevention."

Wu highlights the need to undertake prospective studies to clarify the effect of different classes of antidepressants on risk of fracture. The effect of dose and duration of antidepressant therapy on fracture risk, and how other medications interact with the effect of SSRIs on fracture risk, should be evaluated. "A better understanding of the contributions of the role of cognitive and physical limitations in the relationship between SSRI treatment and risk of fracture is also needed," Wu concludes.

\section{Joana Osório}

Original article $\mathrm{Wu}$, $\mathrm{Q}$. et al. Selective serotonin reuptake inhibitor treatment and risk of fractures: a meta-analysis of cohort and case-control studies. Osteoporosis Int. doi:10.1007/s00198-011-1778-8 